\title{
Decreased Atherogenic Response to Dietary Cholesterol in Pigeons after Stimulation of Cholesterol Catabolism in Early Life
}

\author{
M. T. R. Subbiah, D. Deitemeyer, and R. L. Yunker, Departments of Medicine \\ and Pathology, University of Cincinnati Medical Center, \\ Cincinnati, Ohio 45267
}

\begin{abstract}
A в S T R A C T Cholesterol catabolism was stimulated in 6-wk-old White Carneau pigeons using a laboratory stock diet containing $1.3 \%$ cholestyramine resin. After $8 \mathrm{wk}$ on this diet the animals were returned to control stock diet (no resin) for another 8-wk period. When subsequently challenged with a diet containing $0.5 \%$ cholesterol, cholestyramine-pretreated pigeons exhibited significantly lower serum cholesterol level when compared with controls and this "hyporesponder" behavior persisted throughout the study period. Furthermore, the aorta of cholestyramine-treated animals exhibited significantly $(a)$ lower prevalence and severity of atherosclerosis and $(b)$ lower cholesterol content. These studies demonstrate for the first time that enhancement of cholesterol catabolism in early life improves resistance to diet-induced atherosclerosis in later life in this avian model.
\end{abstract}

\section{INTRODUCTION}

Epidemiological and pathological studies of atherosclerosis have suggested that this disease process starts

This work was presented at the 54th Annual Meeting of the American Heart Association at Dallas, TX, 16-19 November 1981 (15).

Address correspondence to Dr. Subbiah.

Received for publication 29 December 1982 and in revised form 15 February 1983. early in life and develops over a period of several years $(1,2)$. Therefore, it is of primary interest to determine whether interventions started early in life can prevent the age-related progression leading to complicated atherosclerotic lesions. Since high serum cholesterol is a risk factor for atherosclerosis (3), attempts to improve the handling of cholesterol in adult life by manipulating cholesterol metabolism in neonatal life appears to have considerable potential as a means to decrease atherogenic tendency in later years (4). Studies from our laboratory in neonatal guinea pigs showed (5) that enhancement of cholesterol degradation by cholestyramine for the first 6 wk of life improved the ability of these animals to handle cholesterol challenge in adult life. Whether such stimulation of cholesterol catabolism in early life can also improve resistance to dietary cholesterol-induced atherogenesis is not known and this question was investigated in the White Carneau pigeon. This animal model develops spontaneous atherosclerotic lesions (6-8) and when fed cholesterolcontaining diet, the severity of atherosclerosis is markedly accelerated (9). In this communication, we report for the first time that enhancement of cholesterol catabolism during early life improves resistance to dietinduced atherosclerosis in later life.

24 White male Carneau pigeons (6 wk old) from the colony at Palmetto Pigeon Plant, Sumter, SC were randomly assigned to one of the two groups. During 
the first 8 wk (period 1) one group was fed standard laboratory pigeon chow (Ralston Purina Co., St. Louis, $\mathrm{MO})$ and the other group was fed the same pigeon chow but containing 2.5\% Questran (containing $1.3 \%$ cholestyramine resin, Mead Johnson Co., Evansville, IN). After that both groups were fed standard laboratory pigeon chow (free of dietary cholesterol) for an additional $8 \mathrm{wk}$ (period II). Subsequently, they were challenged with a diet containing $\mathbf{0 . 5 \%}$ cholesterol for 8 wk (period III). Blood was drawn by cardiac puncture, (under light nembutal anesthesia), at regular intervals from all the pigeons. Plasma cholesterol levels were measured using the LRC method (10).

At the end of cholesterol feeding period, the pigeons were killed by exsanguination. The aortas were dissected quickly, and the area in between $2-\mathrm{mm}$ points below and above the celiac branch of the aorta (site of spontaneous atherosclerotic lesions [11] in these pigeons) was cut out and placed on top of a transparent plastic sheet lying on graph paper. The aortas were then photographed and the color slides obtained were projected on a screen. The area of raised atheroscle-

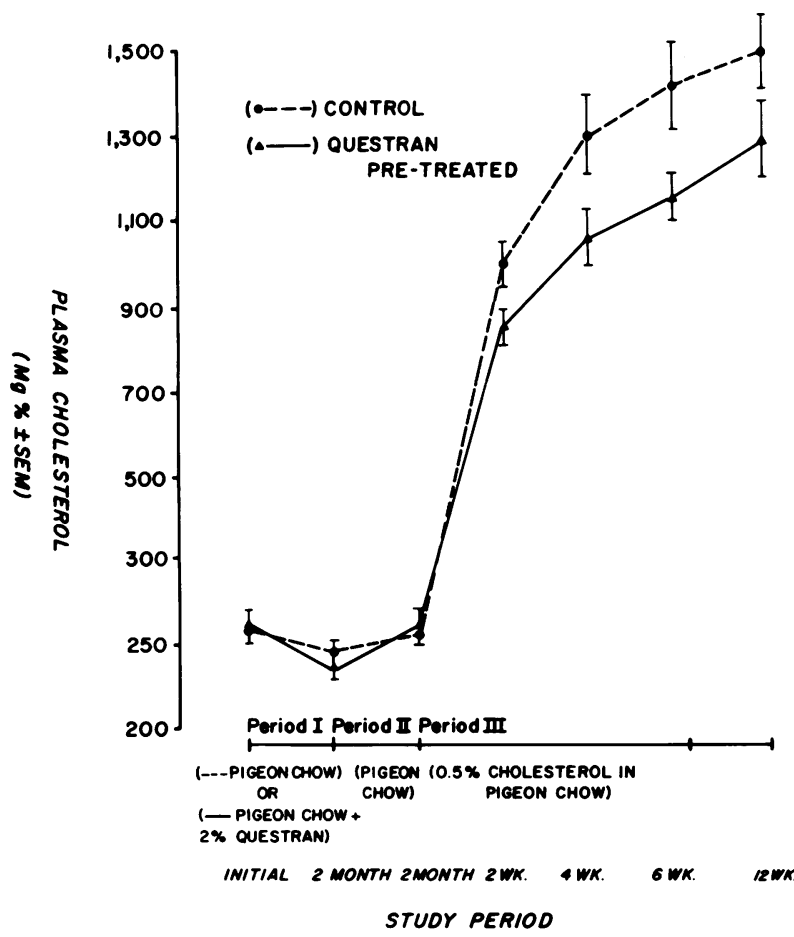

Figure 1 Changes in plasma cholesterol levels in control and cholestyramine-pretreated pigeons during the study. Each point represents the mean value from 9 to 10 animals and the vertical bars represent SEM. Same animals were sampled at various time points. Age-matched pigeons on chow diet throughout had plasma cholesterol of $289 \pm 13 \mathrm{mg} /$ $100 \mathrm{ml}$ at the end of the study. rotic lesion (yellow) was traced on paper and estimated by planimetry, as previously described $(8,11)$. The area of the lesion at the celiac branch of the aorta was used for chemical studies. For light microscopic studies, frozen sections of aorta were cut and stained with oil red $o$ and hematoxylin and eosin stains.

Total lipids from aortas was extracted with $20 \mathrm{vol}$ of chloroform:methanol (2:1), and various lipids were separated by thin-layer chromatography on silica gel using a solvent system of heptane:isopropyl ether:acetic acid (65:40:4, vol/vol/vol), as described previously (10). In this solvent system all the phospholipids remain at the origin. Bands corresponding to standards of free cholesterol and cholesteryl ester were marked and scraped into vials. Both sterols and steryl esters were eluted from the silica gel $\mathrm{G}$ by three extractions, each with $4 \mathrm{ml}$ of chloroform. The extracts were pooled and evaporated to dryness. The steryl ester fraction was hydrolysed with $1 \mathrm{~N} \mathrm{NaOH}$ in $20 \%$ ethanol and the resulting sterols were extracted with hexane. The sterols (both free and esterified) were analyzed by gasliquid chromatography using $5 \alpha$-cholestane as an internal standard $(8,10)$. The sterols were quantitated on the basis of the internal standard peak areas. Sterols were analyzed using a Packard model 409 gas chromatograph (Packard Instrument Co., Inc., Downers Grove, IL) equipped with 4-ft 4-mm i.d. glass columns containing 3\% OV-17 (on gas chrom Q, 100-120 mesh) packings. Column conditions were as follows: oven, $230^{\circ}$; injector, $270^{\circ}$, detector, $300^{\circ}$; and carrier gas, helium $50 \mathrm{ml} / \mathrm{min}$.

TABLE I

Effect of Neonatal Cholestyramine Pretreatment on Atherogenic Response to Dietary Cholesterol Challenge in Adult Life (Mean \pm SEM)

\begin{tabular}{|c|c|c|c|c|}
\hline \multirow[b]{2}{*}{ Group $(n)$} & \multirow[b]{2}{*}{ Age } & \multicolumn{2}{|c|}{ Atherosclerotic placques } & \multirow[b]{2}{*}{ Aortic cholesterol } \\
\hline & & Prevalance & $\begin{array}{c}\text { Surface area } \\
\text { involved }\end{array}$ & \\
\hline & $w k$ & $\%$ & $\%$ & $\begin{array}{l}\mu \mathrm{g} / 100 \mathrm{mg} \\
\text { wet } w \mathrm{t}\end{array}$ \\
\hline \multicolumn{5}{|l|}{ Cholesterol-fed } \\
\hline Control (9) & $34 \pm 1$ & 90 & $13.1 \pm 2.5 \ddagger$ & $493.1 \pm 105.3 \ddagger$ \\
\hline Cholestyramine & & & & \\
\hline pretreated (11) & $34 \pm 1$ & 30 & $5.0 \pm 2.6$ & $157.6 \pm 87.5$ \\
\hline \multicolumn{5}{|l|}{ Chow fed } \\
\hline $\begin{array}{l}\text { Control (10, age } \\
\text { matched) }\end{array}$ & $36 \pm 1$ & $\mathbf{0}$ & 0 & $112.2 \pm 11.4$ \\
\hline
\end{tabular}

- Cholesterol contributed to $98 \%$ of sterols in aorta. The rest was made up of cholestanol (1.9\%) and traces of a peak corresponding to retention time of campesterol.

$\ddagger P<0.05$ for difference between control and cholestyramine fed groups under cholesterol-fed conditions. 


\section{RESULTS}

Cholestyramine feeding for 2 mo did not influence the body weight (in grams) of pigeons (control: 598 \pm 7 , cholestyramine treated: $575 \pm 6$ ). Cholestyramine treatment (period I) caused a slight but significant $(P$ $<0.05$ ) decrease in plasma cholesterol (Fig. 1). Upon cessation of cholestyramine feeding and return to nor- mal chow diet (period II) plasma cholesterol levels returned to control levels. Both control and experimental groups were continued on the control chow diet for a 2-mo period. Subsequently, both groups of animals were challenged with a $0.5 \%$ cholesterol containing chow diet for a 3-mo period (period III). Initially, plasma cholesterol levels increased markedly in both groups of animals (Fig. 1). Subsequently, in cho-
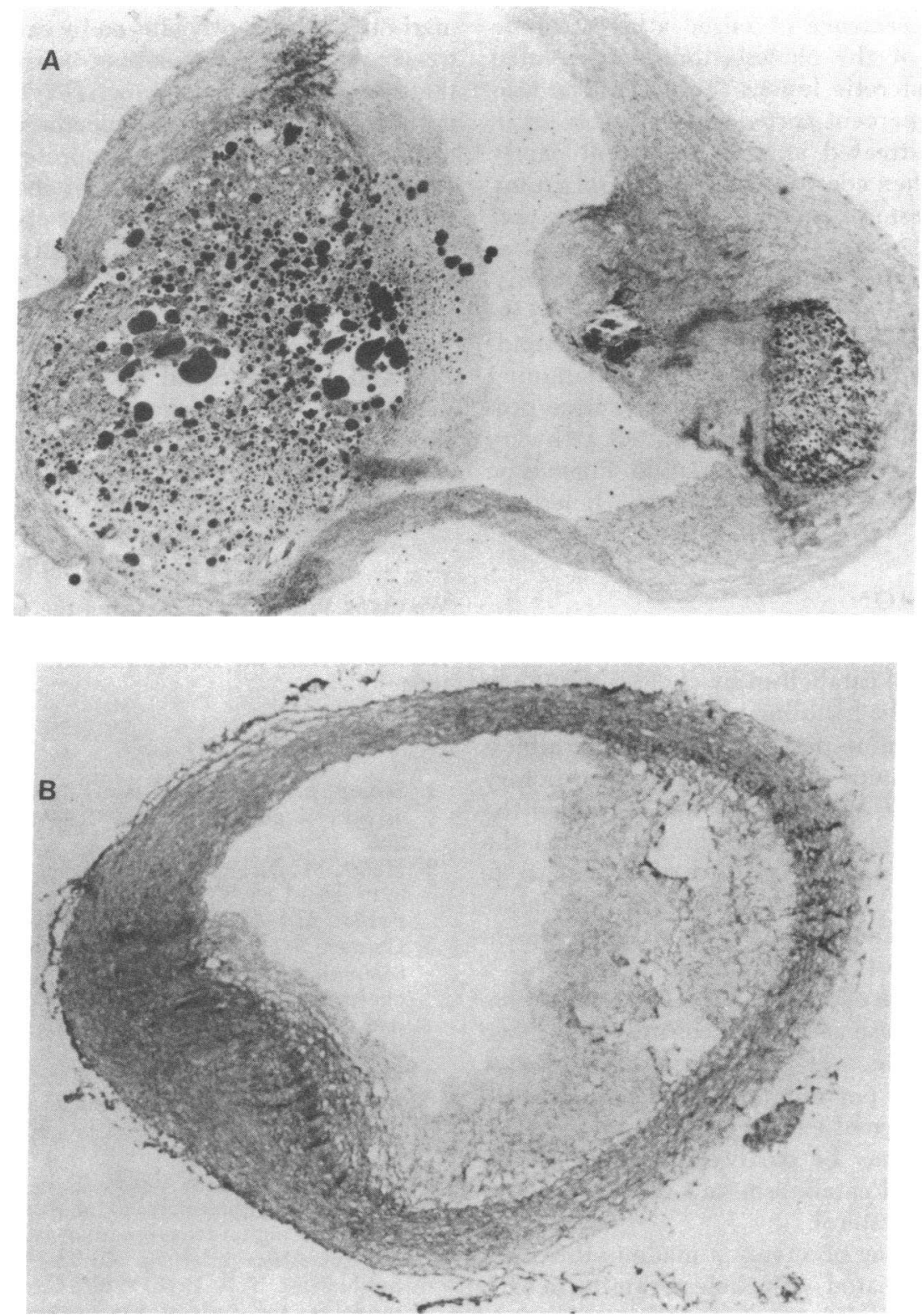

FigURE 2 Micrographs of aortas from control (A) and cholestyramine-pretreated (B) pigeons fed a atherogenic diet. Note marked raise in intima with lipid containing cells surrounded by collagen-containing area in control pigeon, while intima is only slightly raised with a few layers of lipid containing cells in the cholestyramine-pretreated pigeons. (Oil red o stain $\times 40$ ). 
lestyramine-pretreated pigeons, plasma cholesterol levels were significantly lower $(P<0.05)$ when compared to the control group. This "hyporesponder" pattern in plasma cholesterol changes was maintained throughout the study period. Plasma cholesterol of pigeons on chow diet was $287 \pm 13 \mathrm{mg} / 100 \mathrm{ml}$ at this age.

At the end of cholesterol challenge period, the animals were killed and examined for the prevalence and extent of atherosclerosis. While $90 \%$ of the control group showed the presence of raised atherosclerotic lesions, only $30 \%$ of the cholestyramine pretreated group had atherosclerotic lesions (Table I). The atherosclerosis index (percent aortic surface involved) in cholestyramine pretreated animals was significantly lower $(P<0.05)$ when compared to the control group. Furthermore, cholesterol content of aorta (an important indicator of atherogenesis) was significantly lower $(P<0.01)$ in cholestyramine pretreated group. Histological examination of the aorta (Fig. 2A and B) indicated that intima was markedly raised with lipidladen foam cells surrounded by collagen-containing areas in control animals. In the cholestyramine-pretreated pigeon the intima was slightly raised with very moderate collagen and lipid accumulation. Pigeons on chow diet throughout had low aortic cholesterol (Table I).

\section{DISCUSSION}

One of the observations made in this study is that stimulation of cholesterol catabolism by cholestyramine in early life improves the handling of cholesterol in adult life. The cholestyramine-pretreated animals exhibit a hyporesponder behavior in their response to dietary cholesterol challenge. We have further examined the mechanisms of this phenomenon and found that the hepatic activity of cholesterol $7 \alpha$-hydroxylase (rate limiting enzyme of cholesterol catabolism to bile acids), which is stimulated following neonatal treatment with cholestyramine (12), remains persistent up to 2 mo after cessation of cholestyramine treatment (cholesterol $7 \alpha$-hydroxylase activity in controls: $0.68 \pm 0.08$ $\mathrm{pmol} / \mathrm{mg}$ per min, cholestyramine pretreated: $2.56 \pm 0.43 \mathrm{pmol} / \mathrm{mg}$ per $\mathrm{min}, P<0.01$, unpublished data). The persistence of enhanced cholesterol $7 \alpha$-hydroxylase activity may be partly responsible for the increased cholesterol catabolism and subsequent lowering of serum cholesterol.

The most interesting observation made in this study is that the pigeons treated with cholestyramine in early life not only show an improved response in terms of serum cholesterol levels, but also show marked resistance to cholesterol-induced atherosclerosis. The mechanism responsible for the phenomenon is not clear. It is conceivable that some of the effect could be due to the lower plasma cholesterol noted in this study. Although the plasma cholesterol levels in the cholestyramine-pretreated group were lower when compared with controls (Fig. 1), they were nevertheless quite high $(>900 \mathrm{mg} / 100 \mathrm{ml})$ in comparison to normal levels in pigeons $(250 \mathrm{mg} / 100 \mathrm{ml})$. Thus, it appears that in addition to serum cholesterol differences, and increased activity of cholesterol $7 \alpha$-hydroxylase, arterial wall metabolism of cholesterol might be permanently altered by early cholestyramine treatment. In this connection, it is interesting to note that recent studies have shown $(13,14)$ that cholestyramine treatment enhanced specific receptor-mediated catabolism of low density lipoproteins. However, it is not known whether this effect of cholestyramine treatment is also as persistent as that observed for hepatic cholesterol $7 \alpha$-hydroxylase activity in this and previous study (11). Further studies are needed on the persistent changes in arterial lipid metabolism following early cholestyramine treatment, in order to understand the improved resistance to atherosclerosis noted in this study. In summary, our studies show for the first time that manipulation of cholesterol metabolism in early life is effective in influencing atherogenic response in later life in this avian model.

\section{ACKNOWLEDGMENTS}

We thank Wilma Roll for typing the manuscript.

This study was supported in part by grant HL-24071 and HL-24263 from the National Heart, Lung, and Blood Institute.

\section{REFERENCES}

1. Strong, J. P., and H. C. McGill, Jr. 1969. The pediatric aspects of atherosclerosis. J. Atheroscler. Res. 9: 251265.

2. Haust, M. D. 1971. The morphogenesis and fate of potential and early atherosclerotic lesions in man. Hum. Pathol. 2: 1-29.

3. Conner, W. E. 1979. The relationship of hyperlipoproteinemia to atherosclerosis: The decisive role of dietary cholesterol and fat. In The Biochemistry of Atherosclerosis. A. M. Scanu, editor. Marcel Decker, Inc., New York. p. 371-418.

4. Subbiah, M. T. R. 1979. Neonatal manipulation of cholesterol metabolism: A potential maneuver to improve the handling of cholesterol in adult life. Artery. 6: 8288.

5. Li, J. R., L. K. Bale, and M. T. R. Subbiah. 1979. Effect of enhancing cholesterol catabolism during neonatal life of guinea pig on its subsequent response to dietary cholesterol. Atherosclerosis. 32: 93-98.

6. Subbiah, M. T. R. 1976. White Carneau Pigeon: An ideal model for the study of progression and regression of atherosclerosis. Biochem. Exp. Biol. 12: 229-243.

7. Lauper, N. T., K. K. Unni, B. A. Kottke, and J. L. Titus. 1975. Anatomy and pathology of aorta of White Carneau Pigeon. Lab. Invest. 32: 536-551. 
8. Subbiah, M. T. R., K. K. Unni, B. A. Kottke, I. A. Carlo, and D. M. Dinh. 1976. Arterial and metabolic changes during critical period of sterol accumulation in pigeon aorta. Exp. Mol. Pathol. 24: 287-301.

9. Clarkson, T. B., R. W. Pritchard, M. G. Netsky, and H. B. Lofland. 1959. Atherosclerosis in pigeons: Their spontaneous occurance and resemblance to human atherosclerosis. Arch. Pathol. 68: 143-147.

10. LRC Manual of Operations 1979. Vol. 1, Lipid and Lipoprotein Analysis, U. S. Printing Office, Washington, DC, (National Heart, Lung and Blood Institute, DHEW Publications No. 75-028).

11. Subbiah, M. T. R. 1980. Effect of interventions started early in life on the development of spontaneous atherosclerosis. Proc. Soc. Exp. Biol. Med. 165: 32-38.

12. Hassan, A. S., L. S. Gallon, L. S. Zimmer, W. F. Balistreri, and M. T. R. Subbiah. 1980. Persistent enhance- ment of bile acid and synthesis following stimulation of cholesterol catabolism in neonatal life. Steroids. 38: 477486.

13. Slater, H. R., C. J. Packard, S. Bicker, and J. Shepherd 1980. Effect of cholestyramine on receptor mediated plasma clearance and tissue uptake of human low density lipoproteins in the rabbit. J. Biol. Chem. 255: 1021010213.

14. Shepherd, J., C. J. Packard, S. Bicker, T. D. Veitch-Lawrei, and C. H. G. Morgan. 1980. Cholestyramine promotes receptor mediated low density lipoprotein catabolism. N. Engl. J. Med. 302: 1219-1222.

15. Subbiah, M. T. R., D. Deitemeyer, and R. L. Yunker. 1981. Neonatal stimulation of cholesterol catabolism improves resistance to diet induced hypercholesterolemia and atherosclerosis in adult life. Circulation. 64(IV): 112 